\title{
Emergency triage, assessment and treatment at a district hospital in Malawi
}

\author{
Hooi-Ling Harrison, ${ }^{1}$ Nigel Raghunath, ${ }^{2}$ Michele Twomey ${ }^{3}$
}

${ }^{1}$ Emergency Department, College of Emergency Medicine, London, UK

2Emergency Department, Croydon University Hospital, London, CR7 7YE, UK

${ }^{3}$ School of Public Health, University of Cape Town, South Africa

\section{Correspondence to}

Dr Hooi-Ling Harrison, University Hospital Lewisham, Emergency Dept, London, SE13 6LH, UK; hlingharrison@hotmail.co.uk

Accepted 19 August 2011 Published Online First 8 December 2011

\section{ABSTRACT}

Rumphi District Hospital in Northern Malawi had no emergency triage, assessment or treatment system for the over 5 year olds. Eighty healthcare workers were trained on the South African Triage Scale, which was then implemented within a modified outpatient department. Provision of medical equipment and construction of an emergency room took place to allow early life saving treatment.

\section{INTRODUCTION}

Rumphi District Hospital in the Northern Region of Malawi serves a population of 225000 and because of its location near the borders of Zambia the hospital has as many as 314119 attendances to its outpatient department (OPD) each year. ${ }^{1}$ This department, run by three clinicians is responsible for assessing, discharging or admitting patients to a ward similar to that of an emergency department in the UK.

However, there are significant differences: (i) there is no objective triage system in place for patients over the age of 5 years; (ii) patients are seen in time order rather than according to medical urgency; (iii) the large number of patients and lack of human resources lead to delays in recognising those who are medically unwell; (iv) thorough assessment and treatment is not performed owing to lack of equipment, a restricted space and the absence of nurses from the OPD; (v) patients are transferred to a ward without any treatment where they will wait for the sole nurse working on a ward of three patients per bed to attend to them.

These problems of lack of resources and delay in treatment clearly contribute to high morbidity and mortality rates in particular from those diseases common in the developing world-namely, malaria, HIV-related illnesses and tuberculosis, ${ }^{2}$ and to the feeling of frustration and dissatisfaction among healthcare workers. ${ }^{3}$

\section{THE PROJECT}

From observation of these challenges during 1-year of volunteer work as a medical officer with the charity Voluntary Services Overseas three objectives were set to tackle them:

1. To train healthcare workers on an objective triage system to distinguish between patients' medical urgency in the OPD of Rumphi District Hospital.

2. To create an emergency room to conduct life-saving care.

3. To integrate the use of the South African Triage Scale into the current OPD system.
The overall objective was to reduce morbidity and mortality in relation particularly to malaria, HIV/AIDS-related illness and tuberculosis, in accordance with the World Health Organizations' (WHO) goals ${ }^{2}$

\section{Triage}

Triage systems in the developed world are well established in emergency departments. However, Malawi has not had an effective triage system to sort patients over the age of 5 years (for patients under the age of 5 years the WHO Emergency Triage Assessment and Treatment system is in place). The South African Triage Group developed a scale adapting existing models for use in Africa. ${ }^{4-6}$ The scale uses the simple colour coding system of red, orange, yellow and green to determine urgency. Patients are given a triage early warning score according to their physiology, which is then entered into a discriminator list. This acts as a safety net to pick up sick patients not identified with the triage early warning score and gives a final colour code to the patient. ${ }^{4-6}$

South Africans have an average life expectancy of 52. The health needs of the majority are served by an under-resourced public health service, their disease spectrum is mainly made up of communicable diseases-namely, malaria, TB and HIV/ AIDS, and the skill mix of healthcare workers is wide. ${ }^{4}$ In Malawi life expectancy is 49 and the country encounters similar challenges which thus supported the decision to use the South African Triage Scale in the OPD of Rumphi District Hospital. $^{2}$

Eighty healthcare workers were trained on this triage system using lectures, discussion forums and simulation on a 1-day course over a 1-week period by two emergency medicine physicians from the UK (see figure 1). Participants were requested to do a test before and after the course to provide evidence of their ability to triage following completion of the course.

\section{The emergency room}

An emergency room was created by the extension of an existing clinical room (see Figure 3 ). It is equipped with an examination couch, two resuscitation beds, essential resuscitation equipment and additional equipment to provide oxygen, deliver nebulisers, measure glucose and haemoglobin. It does not cater for peripartum mothers, children under 5 or multiply injured trauma patients who go to maternity, paediatric ward and theatre, respectively.

\section{Implementation in the OPD}

One nurse and two patient attendants at a time are allocated to the OPD to perform triage using the 


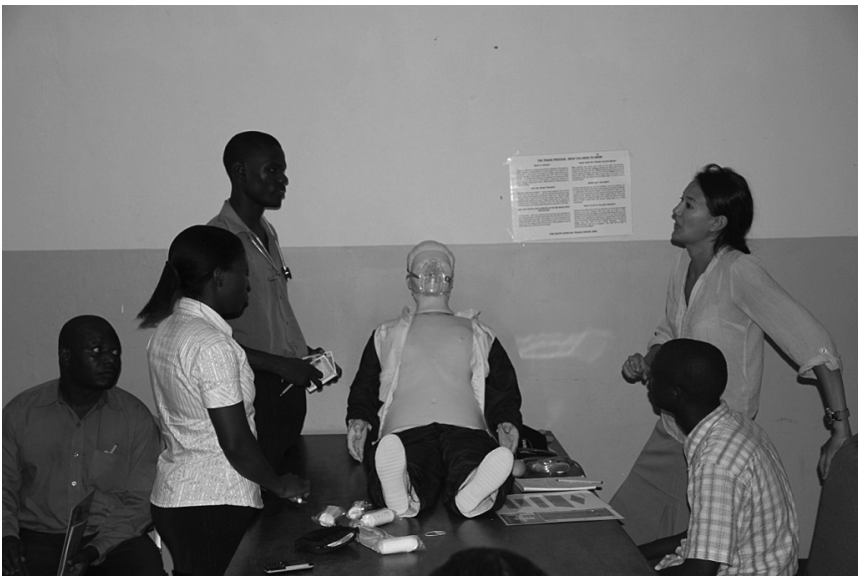

Figure 1 Simulation training.

South African Triage Scale with procured equipment and to initiate treatment in the emergency room. After colour coding, patients are asked to wait on the equivalent colour coded benches to be seen by a clinician, unless they have been triaged as red, in which instance the patient would enter the emergency room for immediate assessment and treatment (see Figure 2).

\section{DISCUSSION}

The whole project took 4 months and now the system is operational. Positive feedback has been received from the healthcare workers who report satisfaction from delivering patient care in a clinically driven order with clear benefit. An additional positive outcome is less pressure on nurses to attend to new patients

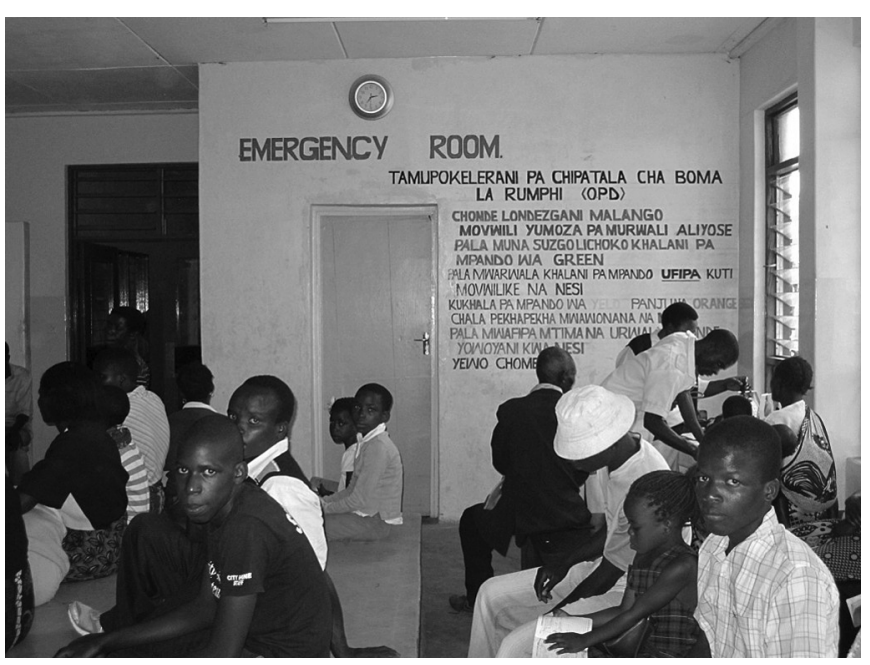

Figure 2 The waiting room where triage is performed.

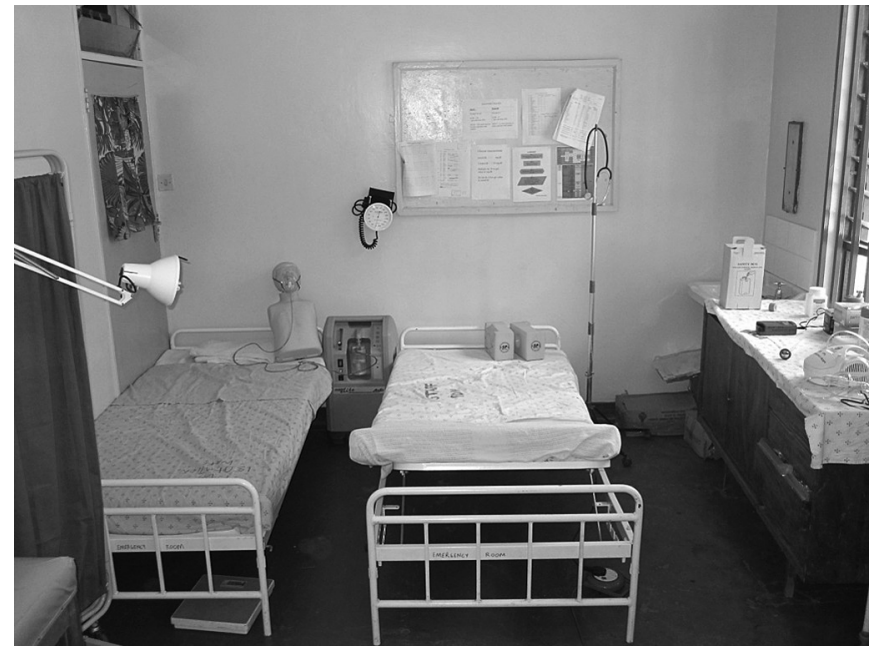

Figure 3 Inside the Emergency room.

owing to the initiation of treatment in the OPD before the patient arrives on the ward.

Data are yet to be collected on morbidity and mortality the causes of which are of course multifactorial; however, objectively it is seen that patients are receiving treatment earlier for life-threatening conditions.

The clinicians and nurses have taken ownership of the project particularly following the training of trainers on the South African Triage Scale which should help to ensure its sustainability. Currently, results are being analysed on the reliability of healthcare workers triage ratings after such training.

Funding The project was provided by Irish Aid, Malawi and Mayday Hospital, London, UK.

\section{Competing interests None.}

Contributors $\mathrm{H}$-LH was responsible for the planning and completion of the project and the writing of this report. NR was involved in the training of health workers, completion of the emergency room and assisted in the writing of the report. MT revised the article before submission.

Provenance and peer review Not commissioned; externally peer reviewed.

\section{REFERENCES}

1. Munthali P. Health Management Information System Bulletin Rumphi District Hospital 2009-2010. Rumhi Hospital, Malawi, 2011:36-7.

2. http://www.who.int/countries/mwi/en/ laccessed 4 Sep 2011).

3. Palmer D. Tackling Malawi's human resources crisis. Reprod Health Matters 2006;14:27-39. http://www.who.int/entity/healthsystems/gf17.pdf laccessed 4 Sep 2011).

4. Bruijns SR, Wallis LA, Burch VC. A prospective evaluation of the Cape Triage Score in the emergency department of an urban public hospital in South Africa. Emerg Med J 2008;25:398-402.

5. Gottschalk SB, Wood D, DeVries S, et al; On behalf of the Cape Triage Group. The cape triage score: a new triage system South Africa: Proposal from the cape triage group. Emerg Med J 2006;23:149-53.

6. Wallis LA, Twomey M. Workload and casemix in Cape Town emergency departments. S Afr Med J 2007;97:1276-80. 